7-2016

\title{
Consequences of the Angular Spectrum Decomposition of a Focused Beam, including Slower than C Beam Propagation
}

Gérard Gouesbet

Normandie Université, gouesbet@coria.fr

James A. Lock

Cleveland State University, j.lock@csuohio.edu

Follow this and additional works at: https://engagedscholarship.csuohio.edu/sciphysics_facpub

Part of the Physics Commons

How does access to this work benefit you? Let us know!

\section{Repository Citation}

Gouesbet, Gérard and Lock, James A., "Consequences of the Angular Spectrum Decomposition of a Focused Beam, including Slower than C Beam Propagation" (2016). Physics Faculty Publications. 389.

https://engagedscholarship.csuohio.edu/sciphysics_facpub/389

This Article is brought to you for free and open access by the Physics Department at EngagedScholarship@CSU. It has been accepted for inclusion in Physics Faculty Publications by an authorized administrator of EngagedScholarship@CSU. For more information, please contact library.es@csuohio.edu. 


\title{
Consequences of the angular spectrum decomposition of a focused beam, including slower than $c$ beam propagation
}

\author{
Gérard Gouesbet ${ }^{a, *}$, James A. Lock
}

Keywords:

Speed of light

Optical theorem

Angular spectrum decomposition

Generalized Lorenz-Mie theory

\begin{abstract}
A B S T R A C T
When dealing with light scattering and propagation of an electromagnetic beam, there are essentially two kinds of expansions which have been used to describe the incident beam (i) a discrete expansion involving beam shape coefficients and (ii) a continuous expansion in terms of an angular spectrum of plane waves. In this paper, we demonstrate that the angular spectrum decomposition readily leads to two important consequences, (i) laser light beams travel in free space with an effective velocity that is smaller than the speed of light $c$, and (ii) the optical theorem does not hold for arbitrary shaped beams, both in the case of electromagnetic waves and scalar waves, e.g. quantum and acoustical waves.
\end{abstract}

\section{Historical background}

Physicists (and philosophers) have recurrently been worried about the existence of "actual" infinities, "actual" being taken in the sense of Aristotle; that is to say in contrast with "potential" infinities. A number of examples of this are as follows. Epicurus (341-270 B.C.), an atomist scientist and Greek philosopher stated that any "atom" has the same velocity as any other "atom", more specifically that heavy "atoms" possess the same velocities as lighter ones, at least when their motion is not modified by any collision [1]. According to the concept of "atom", usually attributed to Leucippus (circa 460-370 B.C.), atoms are indivisible physical entities (in the greek language "atomos" means indivisible) which can be combined to form aggregates of matter. This hypothesis was repeated by one on his followers, namely Lucretius (circa 98-55 B.C.), who stated in his famous De Rerum Natura that "all atoms in vacuum possess the same velocity, independently of their

\footnotetext{
* Corresponding author.

E-mail address: gouesbet@coria.fr (G. Gouesbet).
}

different weights" [2]. Epicurus is also the author of another statement having a very modern flavor, namely that the velocity of images or "projections" that allow us to see the objects is the greatest velocity attainable. In modern language, the speed of light, denoted by the letter $c$, is the greatest possible speed (and we may understand from Epicurus that it is finite). Jumping over the millennia, Newton's theory of gravitation implied action at a distance. But the fact that it would happen with an infinite speed made him apparently concerned although as he said "hypotheses non fingo" (I contrive no hypotheses). Newton's mechanics, with its action at a distance with an infinite speed, was in deep opposition to Descartes' mechanics which rejected the possibility of action at distance. Descartes did not use the concept of forces, and produced a kinematic description of the world in which movements are transmitted by direct contacts within a substance carrying out eddies [3]. Descartes' mechanics was more satisfactory than Newton's insofar as it did not use the idea of an action at distance. However it was rejected by the verdict imposed by experiments, leaving us before the advent of quantum field theory with forces acting instantaneously at a distance. 
Returning to the story of light, it has been known since the measurements by Romer in 1676 that the speed of light in free space is finite. Einstein took the finiteness of the speed of light as a fundamental principle since he believed that an infinite speed cannot have any sense for any reasonable man [4]. From an epistemological point of view, this is a very strong statement. It meant that for Einstein, the idea of a finite speed of light is clear and distinct in the mind, in the sense of Cartesian rationalism [5]. Such a statement, clear and distinct to the mind, is a priori in the sense of Kant [6], since it is hypothesized before any experimental fact. It is called a first principle. It follows from the first postulate of special relativity that the speed of light in free space is a constant, independent of the motion of the emittor or of the detector. This has the flavor of a first principle that in this paper we shall call the first principle. Although not explicitly stated, the first principle contains the statement that light propagates along straight lines. This a priori status is likely the reason why Einstein did not refer to the experiments of Michelson and Morley in his 1905 special relativity paper. No experiments were required to build an a priori theory, although they are required to corroborate it. The connection with Newton's gravitation and reluctance against the infinite speed of propagation of gravitation was made later in the framework of the general theory of relativity, which states that the speed of propagation of gravitation is the same as the speed of light.

A non-singularity principle (NSP), viewed as a first principle, tells us that a local infinity in physics is not admissible, that is to say: nature (locally) abhors infinity. This first principle has recently been used to propose hypotheses on the a priori (in the sense of Kant) rational (in the sense of Descartes) necessity of quantum mechanics [7,8]. However, the NSP is not viewed as a definitive principle. In agreement with a pyrrhonean statement from Quine's epistemology, any first principle may possess a provisional character, i.e. to be revisable $[9,10]$. This revisability property of Quine's epistemology is illustrated in the present paper by revising Einstein's first principle, namely we shall demonstrate that a transversely localized light beam propagates in free space slower than the speed of light.

Concerning light beams, let us mention that the fundamental characteristic of a transversely localized light beam, considered in the present paper, that distinguishes it from other electromagnetic waves such as radiation produced in the decay of an atomic or molecular excited state, or radiation by an antenna in the long-wavelength limit, is that a beam propagates in one direction, or in a narrow cone of directions, while radiation by an atom or an antenna in the long-wavelength limit is reasonably close to being isotropic. For the main part of the paper, we also do not consider a beam propagating through a material of refractive index $m>1$, where the beam speed is reduced by the refractive index. Nor do we consider the propagation of electromagnetic waves confined within a waveguide or an optical fiber, where the confinement produced by the waveguide walls constrains the propagation speed of the fields to be less than $c$ [11].
As a corollary we shall provide a simple explanation for the failure of the optical theorem, both for electromagnetic waves and for scalar waves (quantum and acoustical waves, e.g. [12,13]. The paper is organized as follows. Section 2 provides a background in light propagation and light scattering. Section 3 deals with the revision of the first principle. Section 4 deals with the optical theorem. Finally, Section 5 is a conclusion.

\section{Beam descriptions}

Generalized Lorenz-Mie theory (GLMT) describes the electromagnetic interaction of an electromagnetic arbitrary shaped beam (ASB), e.g. a laser beam, with a homogeneous spherical particle defined by its diameter and complex refractive index [14]. In GLMT, one of the most important problems concerns the expression of the illuminating ASB. There are essentially two possibilities for this description.

The first possibility (here called the discrete expansion approach) uses the fact that in spherical coordinates the electric and magnetic fields $\mathbf{E}$ and $\mathbf{H}$ (respectively) of an arbitrary (harmonic) shaped beam, with the timedependence of the form $\exp (+i \omega t)$, can be expanded in terms of vector spherical wave functions (VSWFs) according to:

$\mathbf{E}=\sum_{n=1}^{\infty} \sum_{m=-n}^{+n}\left[a_{m n} \mathbf{M}_{m n}^{(1)}(k \mathbf{r})+b_{m n} \mathbf{N}_{m n}^{(1)}(k \mathbf{r})\right]$

$\mathbf{H}=\sum_{n=1}^{\infty} \sum_{m=-n}^{+n} i\left[b_{m n} \mathbf{M}_{m n}^{(1)}(k \mathbf{r})+a_{m n} \mathbf{N}_{m n}^{(1)}(k \mathbf{r})\right]$

in which $\mathbf{M}_{m n}^{(1)}$ and $\mathbf{N}_{m n}^{(1)}$ are VSWFs of the first type, $a_{m n}$ and $b_{m n}$ are expansion coefficients, $k$ is the wave-number and $\mathbf{r}$ is the position vector (e.g. $[15,16]$ for the first presentations of such expansions in a GLMT framework, in particular Eqs. (10) and (11) in [15]). We do not need to specify the expressions of the VSWFs here, but see [17] for details. In the original formulation of GLMT, the field expansions were provided in an equivalent way that relied on Bromwich scalar potentials (see [14] and references therein), and introduced two sets of beam shape coefficients (BSCs) $g_{n T M}^{m}$ and $g_{n T E}^{m}$, which are defined in such a way so as to exhibit a simple appealing form in the case of an axisymmetric beam, in particular a plane wave [18]. The BSCs are proportional to the expansion coefficients $a_{m n}$ and $b_{m n}$ of Eqs. (1) and (2) according to [19]:

$a_{m n}=-i k E_{0} c_{n}^{p w}(-1)^{m}(-1)^{(m-\mid m) / 2} \frac{(n-m) !}{(n-|m|) !} g_{n, T E}^{m}$

$b_{m n}=k E_{0} c_{n}^{p w}(-1)^{m}(-1)^{(m-|m|) / 2} \frac{(n-m) !}{(n-|m|) !} g_{n, T M}^{m}$

in which the coefficients $c_{n}^{p w}$ (" $p w$ " standing for "plane wave") appearing in the Bromwich formulation of LorenzMie theory are [20]:

$c_{n}^{p w}=\frac{(-i)^{n+1}}{k} \frac{2 n+1}{n(n+1)}$ 
In some cases, in particular for an on-axis Gaussian beam $[18,21]$, the double set of coefficients reduces to a single set of special coefficients denoted by $g_{n}$. For further use, when we discuss the optical theorem in Section 4, we also mention that from energy balance, it can be shown that the extinction cross-section of the scattering process is given by [14,21]:

$$
\begin{aligned}
C_{e x t}= & \frac{4 \pi}{k^{2}} \operatorname{Re}\left[\sum _ { n = 1 } ^ { \infty } \sum _ { m = - n } ^ { + n } \frac { 2 n + 1 } { n ( n + 1 ) } \frac { ( n + | m | ) ! } { ( n - | m | ) ! } \left(a_{n}\left|g_{n, T M}^{m}\right|^{2}\right.\right. \\
& \left.\left.+b_{n}\left|g_{n, T E}^{m}\right|^{2}\right)\right]
\end{aligned}
$$

When the reduction of the double set of BSCs to a single set of special BSCs is feasible, Eq. (6) reduces to [21,22]:

$$
C_{e x t}=\frac{2 \pi}{k^{2}} \operatorname{Re} \sum_{n=1}^{\infty}(2 n+1)\left|g_{n}\right|^{2}\left(a_{n}+b_{n}\right)
$$

The second possibility for the description of an ASB (here called the angular spectrum decomposition) relies on the use of a decomposition in terms of plane waves having the same wave length but different propagation directions. This decomposition introduces two vector spectral functions of $k_{x}$ and $k_{y}$ in Cartesian coordinates, the electric function $\mathbf{S}_{i}^{E}$ and the magnetic function $\boldsymbol{S}_{i}^{H}, i=x, y, z$. We may choose two spectral components as being independent, e.g. $S_{x}^{E}\left(k_{x}, k_{y}\right)$ and $S_{y}^{E}\left(k_{x}, k_{y}\right)$ which are defined using Fourier transforms according to:

$$
\begin{aligned}
\left.k^{2} \quad \begin{array}{l}
E_{x}(x, y, 0) \\
E_{y}(x, y, 0)
\end{array}\right)= & \int_{-\infty}^{+\infty} \int_{-\infty}^{+\infty}\left(\begin{array}{l}
S_{x}^{E}\left(k_{x}, k_{y}\right) \\
S_{y}^{E}\left(k_{x}, k_{y}\right)
\end{array}\right) \\
& x \exp \left[i\left(k_{x} x+k_{y} y\right)\right] d k_{x} d k_{y}
\end{aligned}
$$

in which $E_{x}(x, y, 0)$ and $E_{y}(x, y, 0)$ are the transverse components of the electric field in the plane $z=0$, in which $z$ is taken as the forward propagation direction of the ASB. The definition of Eq. (8) follows from $[23,24]$, in agreement with the formalism provided by Goodman [25]. Note also that there are a number of different conventions for the normalization of the Fourier transform and its inverse. In Eq. (8), we follow the convention of $[23,24]$ (see also [26, p. 22]). The inverse Fourier transform reads:

$$
\begin{aligned}
\left(\begin{array}{l}
S_{x}^{E}\left(k_{x}, k_{y}\right) \\
S_{y}^{E}\left(k_{x}, k_{y}\right)
\end{array}\right)= & \left.\frac{1}{4 \pi^{2}} \int_{-\infty}^{+\infty} \int_{-\infty}^{+\infty} k^{2} \begin{array}{l}
E_{x}(x, y, 0) \\
E_{y}(x, y, 0)
\end{array}\right) \\
& \times \exp \left[-i\left(k_{x} x+k_{y} y\right)\right] d x d y
\end{aligned}
$$

Once the spectral components $S_{x}^{E}\left(k_{x}, k_{y}\right)$ and $S_{y}^{E}\left(k_{x}, k_{y}\right)$ are determined, all the other spectral components, namely $S_{z}^{E}\left(k_{x}, k_{y}\right)$ and the magnetic spectral vector $\mathbf{S}_{i}^{H}, i=\chi, y, z$, are determined by using Maxwell's equations [17]. Then, because the propagation of the fields satisfies the Helmholtz equation, we can establish:

$$
\begin{aligned}
k^{2}\left(\begin{array}{c}
E_{x}(x, y, z) \\
E_{y}(x, y, z) \\
E_{z}(x, y, z)
\end{array}\right)= & \int_{-\infty}^{+\infty} \int_{-\infty}^{+\infty}\left(\begin{array}{c}
S_{x}^{E}\left(k_{x}, k_{y}\right) \\
S_{y}^{E}\left(k_{x}, k_{y}\right) \\
S_{z}^{E}\left(k_{x}, k_{y}\right)
\end{array}\right) \\
& \times \exp \left[i\left(k_{x} x+k_{y} y+k_{z} z\right)\right] d k_{x} d k_{y}
\end{aligned}
$$

which is the complete generalization of Eq. (8), with a similar equation for the magnetic field.

A second step in the angular spectrum decomposition relies on the expansion of each plane wave in the spectrum in terms of VSWFs $[17,27]$ then leading to a formulation equivalent to Eqs. (1) and (2). More specifically, the BSCs of the shaped beam are obtained by a superposition of the BSCs of the individual plane wave components of the angular spectrum [27,28]. Indeed, in cases where this can be carried out analytically, the use of the two step process of the angular spectrum approach is very appealing.

\section{Average speed of laser beams}

\subsection{General considerations}

In order to motivate the calculation of the next section, we briefly describe a number of different beam situations of increasing complexity. First, we consider a single plane wave of wavelength $\lambda$, wavenumber $k=2 \pi / \lambda$, angular frequency $\omega=c k$, electric field strength $E_{0}$ traveling in the positive $z$ direction and polarized in the $x$ direction. One has:

$\mathbf{E}=E_{0} \exp [i(k z-\omega t)] \mathbf{u}_{x}$

$\mathbf{H}=\frac{E_{0}}{\mu_{0} c} \exp [i(k z-\omega t)] \mathbf{u}_{y}$

where $\mu_{0}$ is the permeability of free space. It is evident that the plane wave has the velocity $c$ in the positive $z$ direction.

Now consider two plane waves that have both the same wavelength and the field strength, and are both polarized in the $x$ direction. They propagate in the $y z$ plane at the angles $\theta_{k}$ and $-\theta_{k}$ with respect to the positive $z$-axis. They have:

$$
\begin{aligned}
& \mathbf{k}_{1}=k\left[\sin \left(\theta_{k}\right) \mathbf{u}_{y}+\cos \left(\theta_{k}\right) \mathbf{u}_{z}\right] \\
& \mathbf{k}_{2}=k\left[-\sin \left(\theta_{k}\right) \mathbf{u}_{y}+\cos \left(\theta_{k}\right) \mathbf{u}_{z}\right]
\end{aligned}
$$

They superpose to form a fringe pattern with:

$\mathbf{E}_{\text {total }}=\mathbf{E}_{1}+\mathbf{E}_{2}=2 E_{0} \cos \left[k y \sin \left(\theta_{k}\right)\right] \exp \left[i k z \cos \left(\theta_{k}\right)-i \omega t\right] \mathbf{u}_{x}$

$$
\begin{aligned}
\mathbf{H}_{\text {total }}= & \mathbf{H}_{1}+\mathbf{H}_{2}=2 \frac{E_{0}}{\mu_{0} c} \cos \left(\theta_{k}\right) \cos \left[k y \sin \left(\theta_{k}\right)\right] \\
& \times \exp \left[i k z \cos \left(\theta_{k}\right)-i \omega t\right] \mathbf{u}_{y}-2 i \frac{E_{0}}{\mu_{0} c} \sin \left(\theta_{k}\right) \sin \left[k y \sin \left(\theta_{k}\right)\right] \\
& \times \exp \left[i k z \cos \left(\theta_{k}\right)-i \omega t\right] \mathbf{u}_{z}
\end{aligned}
$$

and the Poynting vector is:

$\operatorname{Re}\left(\mathbf{E}_{\text {total }}^{*} \times \mathbf{H}_{\text {total }}\right)=4 \frac{E_{0}^{2}}{\mu_{0} c} \cos \left(\theta_{k}\right) \cos ^{2}\left[k y \sin \left(\theta_{k}\right)\right] \mathbf{u}_{z}$

Since the projection of the wave-vector of each of the plane waves in the positive $z$ direction is $k \cos \left(\theta_{k}\right)$, the group velocity of the composite fields in the positive $z$ direction is $c \cos \left(\theta_{k}\right)$. 
Now consider constructing a zero-order Bessel beam by superposing an infinite number of plane waves whose wave-vectors lie on the surface of a cone making the angle $\theta_{k}$ with the positive $z$ axis. The aplanatic version of the beam fields in cylindrical coordinates $(\rho, \varphi, z)$ is [27,29]:

$$
\begin{aligned}
\mathbf{E}_{x}= & \left\{\frac{1}{2}\left[1+\cos \left(\theta_{k}\right)\right] J_{0}\left[k \rho \sin \left(\theta_{k}\right)\right]\right. \\
& \left.+\frac{1}{2}\left[1-\cos \left(\theta_{k}\right)\right] \cos (2 \varphi) J_{2}\left[k \rho \sin \left(\theta_{k}\right)\right]\right\} \\
& \times \exp \left[i k z \cos \left(\theta_{k}\right)-i \omega t\right\rceil
\end{aligned}
$$

$\mathbf{E}_{y}=\frac{1}{j}\left[1-\cos \left(\theta_{k}\right)\right] \sin (2 \varphi) J_{2}\left[k \rho \sin \left(\theta_{k}\right)\right] \exp \left[i k z \cos \left(\theta_{k}\right)-i \omega t\right]$

$$
\mathbf{E}_{z}=-i \sin \left(\theta_{k}\right) \cos (\varphi) J_{1}\left[k \rho \sin \left(\theta_{k}\right)\right]
$$

with similar expressions for the components of the magnetic field, and where $J_{0}, J_{1}$ and $J_{2}$ are Bessel functions. Evidently, since each constituent plane wave has the component of its wave-vector $k \cos \left(\theta_{k}\right)$ in the positive $z$ direction, the group velocity of the Bessel beam in the positive $z$ direction is again $c \cos \left(\theta_{k}\right)$.

Lastly, consider a beam propagating in the positive $z$ direction whose Gaussian profile in the $z=0$ plane is:

$$
\begin{aligned}
& \left.\mathbf{E}(x, y, 0)=E_{0} \exp -\frac{\rho^{2}}{w_{0}^{2}}\right) \exp (-i \omega t) \mathbf{u}_{x} \\
& \left.\mathbf{H}(x, y, 0)=\frac{E_{0}}{\mu_{0} c} \exp -\frac{\rho^{2}}{w_{0}^{2}}\right) \exp (-i \omega t) \mathbf{u}_{y}
\end{aligned}
$$

Assuming that the beam propagates to $z>0$ via Fresnel diffraction, one has:

$\left.\mathbf{E}(x, y, z)=\frac{E_{0}}{D} \exp -\frac{\rho^{2}}{D w_{0}^{2}}\right) \exp (i k z-i \omega t) \mathbf{u}_{x}$

$\left.\mathbf{H}(x, y, z)=\frac{E_{0}}{\mu_{0} c D} \exp -\frac{\rho^{2}}{D w_{0}^{2}}\right) \exp (i k z-i \omega t) \mathbf{u}_{y}$

where:

$D=1+\frac{2 i z}{k w_{0}^{2}}$

This freely diffracting beam satisfies the paraxial wave equation, also known as the transverse diffusion equation, but not Maxwell's equations. Thus it is not an exact electromagnetic beam, as are the beams of Eqs. (11), (12), (15), (16), (18) and (19). The form of Eqs. (23) and (24) makes it superficially appear as if the beam propagates with the speed $c$ in the positive $z$ direction, since the fields are proportional to $\exp (i k z-\omega t)$. But this is not the case. Consider building the beam of Eqs. (23) and (24) starting with an angular spectrum of plane waves. The plane wave constituent in the $\left(\theta_{k}, \varphi_{k}\right)$ direction has a wave-vector making the angle $\theta_{k}$ with respect to the positive $z$-axis so that its fields are proportional to $\exp \left[i k z \cos \left(\theta_{k}\right)-i \omega t\right)$. When one uses the weighting coefficient:

$A\left(\theta_{k}, \varphi_{k}\right)=A_{0} \exp \left[-k^{2} w_{0}^{2} \sin ^{2}\left(\theta_{k}\right) / 4\right.$

the integral over $\theta_{k}$ in the spectrum may be approximately evaluated when $\theta_{k}$ is assumed to be small for a paraxial beam. This produces the $2 i z /\left(k w_{0}^{2}\right)$ dependence of $D$ in Eq. (25), thus hiding the slower-than- $c$ behavior of the beam in this factor, while leaving the $\exp (i k z-i \omega t)$ dependence explicit. This continues to be the case if one uses the Davis-Barton procedure of adding a series of progressively smaller correction terms to Eqs. (23) and (24) that make the new equations come closer to satisfying Maxwell's equations than Eqs. (23) and (24) did [30,31]. Thus when inquiring about the group velocity of a beam, the most appropriate representation to do so is the angular spectrum representation rather than the coordinate space representation. This is the approach taken in the next section.

\subsection{Plane wave decomposition of a focused Gaussian beam}

As a quantitative example of the angular spectrum decomposition of a beam, we consider a crude approximation to a Gaussian beam, whose non-spreading fields are given by, see Eqs. (21) and (22):

$\left.\mathbf{E}(x, y, z)=E_{0} \exp -\frac{\rho^{2}}{w_{i n c}^{2}}\right) \exp (i k z-i \omega t) \mathbf{u}_{x}$

$\left.\mathbf{H}(x, y, z)=\frac{E_{0}}{\mu_{0} c} \exp -\frac{\rho^{2}}{w_{i n c}^{2}}\right) \exp (i k z-i \omega t) \mathbf{u}_{y}$

Despite the crude nature of the approximation, this beam description does a reasonable job of describing the fields of a Gaussian laser beam in the $\lambda \ll 2 \pi w_{\text {inc }}$ limit. Hereafter it will be convenient, without any loss of generality, to assume that $E_{0}=1$. We note that this beam is tube-like. Wave-fronts are planes perpendicular to the direction of propagation $z$, and the rays, taken as being perpendicular to the wave fronts, are straight lines parallel to the $z$-direction.

This beam is now incident on and focused by a lens of focal length $f$. The focused beam has a converging region, followed by the focal waist region, followed by a diverging region. In the vicinity of the center of the focal waist region, the wave fronts are planar and the magnitude of the electric field may be written as:

$\left.E=\exp \frac{-\rho^{2}}{w_{\min }^{2}}\right)$

in which $w_{\min }$ is the beam waist radius (denoted by $w_{0}$ in GLMT). The incident and beam waist radii for a Gaussian beam focused by a lens are related by [25]:

$w_{\min }=\frac{2 f}{k w_{\text {inc }}}$

as long as $w_{\text {inc }} / f \ll 1$.

The angular spectrum of the focused beam is the Fourier transform of its electric field in the focal plane, i.e. at $z=0$. Using Eq. (9), one obtains:

$S\left(k_{x}, k_{y}\right)=\int_{-\infty}^{+\infty} d x \int_{-\infty}^{+\infty} d y \exp \left[\frac{-\left(x^{2}+y^{2}\right)}{w_{\min }^{2}}\right]$ 


$$
x \exp \left[-i\left(k_{x} x+k_{y} y\right)\right]=\pi w_{\min }^{2} \exp \left[\frac{-\left(k_{x}^{2}+k_{y}^{2}\right) w_{\min }^{2}}{4}\right]
$$

Let us consider a plane wave of wave vector $\mathbf{k}$ in the angular spectrum. It makes an angle $\theta_{k}$ with the forward propagation direction $z$ of the beam and possesses a speed equal to $c$ along its own direction of propagation. Let $\rho_{k}$ be defined as the component of $\mathbf{k}$ perpendicular to the direction $z$ :

$\rho_{k}=k \sin \theta_{k}$

In the paraxial approximation, the velocity component of this plane wave along the forward direction of the beam is then:

$\left.v_{e f f}=c \cos \theta_{k}=c \sqrt{1-\frac{\rho_{k}^{2}}{k^{2}}} \approx c \quad 1-\frac{\rho_{k}^{2}}{2 k^{2}}\right)$

An average velocity of the beam (or effective velocity) may be defined as:

$\left.<v_{e f f}>=c \quad 1-\frac{<\rho_{k}^{2}>}{2 k^{2}}\right)$

where the average of $\rho_{k}^{2}$ over the beam spectrum is given by:

$$
\begin{aligned}
<\rho_{k}^{2}>= & \frac{\int_{-\infty}^{+\infty} d k_{x} \int_{-\infty}^{+\infty} d k_{y}\left(k_{x}^{2}+k_{y}^{2}\right) \exp \left[\frac{-\left(k_{x}^{2}+k_{y}^{2}\right) w_{\min }^{2}}{2}\right]}{\int_{-\infty}^{+\infty} d k_{x} \int_{-\infty}^{+\infty} d k_{y} \exp \left[\frac{-\left(k_{x}^{2}+k_{y}^{2}\right) w_{\min }^{2}}{2}\right]} \\
= & \frac{2}{w_{\min }^{2}}
\end{aligned}
$$

Therefore, from Eqs. (34), (35) and (30), we obtain:

$$
\left.<v_{e f f}>=c \quad 1-\frac{w_{i n c}^{2}}{4 f^{2}}\right)
$$

As was mentioned in Section 3.1, the fact that we are using an approximation to the exact beam fields is not the source of the fact that $\left\langle v_{\text {eff }}\right\rangle<c$.

\subsection{Ray tracing analysis for a focused Gaussian beam}

In this subsection we briefly derive the average speed of the beam discussed in Section 3.2 using ray tracing methods (see [13]). A typical paraxial light ray for the beam of Eqs. (27) and (28) at distance $\rho_{i n c}$ from the $z$-axis propagates in the $z$ direction. As before, this beam is incident on a lens of focal length $f$. After being refracted by the lens, the ray propagates in a straight line at an angle $\theta$ with respect to the $z$ direction, it passes through the focal point, and it afterward continues propagating at the angle $\theta$. One has:

$\tan \theta=\frac{\rho_{\text {inc }}}{f} \approx \theta$ for any paraxial ray such that $\rho_{\text {inc }} \ll f$. We then have:

$\left.v_{\text {eff }}=c \cos \theta \approx c \quad 1-\frac{\rho_{\text {inc }}^{2}}{2 f^{2}}\right)$

for that ray. One can define an average velocity similarly as in Eq. (34), with $\left\langle\rho_{i n c}^{2}\right\rangle$ being the average distance squared of a ray in the beam of Eqs. (27) and (28) from the $z$ axis:

$$
\begin{aligned}
<\rho_{i n c}^{2}> & =\frac{\int_{-\infty}^{+\infty} d x \int_{-\infty}^{+\infty} d y\left(x^{2}+y^{2}\right) \exp \left[\frac{-2\left(x^{2}+y^{2}\right)}{w_{i n c}^{2}}\right]}{\int_{-\infty}^{+\infty} d x \int_{-\infty}^{+\infty} d y \exp \left[\frac{-2\left(x^{2}+y^{2}\right)}{w_{i n c}^{2}}\right]} \\
& =\frac{w_{i n c}^{2}}{2}
\end{aligned}
$$

Then, again using Eq. (30), we readily obtain:

$$
\left.<v_{e f f}>=c \quad 1-\frac{w_{i n c}^{2}}{4 f^{2}}\right)
$$

which agrees with Eq. (36).

\subsection{Curved ray tracing analysis for a focused Gaussian beam}

Expanding on the idea of light rays, we now consider a freely diffracting Gaussian beam whose electric field in the focal plane is given by Eq. (29) of [32]. As in [14,33], this has also been called the $\mathrm{L}$ approximation to a focused Gaussian beam. The surfaces of constant phase of such a beam are given by:

$$
\phi(z, \rho)=k z+\frac{2 \rho^{2} z}{\left.k w_{\min }^{4} 1+\frac{4 z^{2}}{k^{2} w_{\min }^{4}}\right)}-\arctan \frac{2 z}{k w_{\min }^{2}},
$$

where the last term in the right-hand side of Eq. (41) is known as the Gouy phase.

We consider the trajectories of a family of geometrical light rays to be everywhere orthogonal to $\phi(z, \rho)$, as is also the case for electric field lines and equipotentials in electrostatics, and the velocity potential and stream function in aerodynamics. The slope of the paraxial ray trajectories (see p. 352-3 of [34]) is then to the lowest order:

$$
\frac{\partial z}{\partial \rho}=\frac{\partial \phi / \partial \rho}{\partial \phi / \partial z} \approx \frac{4 \rho z}{\left.k^{2} w_{\min }^{4} 1+\frac{4 z^{2}}{k^{2} w_{\min }^{4}}\right)}
$$

The solution of this differential equation is:

$\rho(z)=\rho_{0} \sqrt{1+\frac{4 z^{2}}{k^{2} w_{\text {min }}^{4}}}$

in which $\rho_{0}$ is $\rho(0)$ (see [35]). These ray paths are a set of hyperbolas, being asymptotic to the straight ray paths described in Section 3.3 far from the focal waist and propagating in exactly the $z$ direction in the focal plane. A similar set of curved ray paths for diffraction by a straight edge are shown on p. 577 of [36]. 
We assume that light travels with the speed $c$ along these curved ray paths. Let us consider two planes perpendicular to the direction $z$, located at $z=-L$ and $z=+L$. The time $T$ required along a trajectory to travel from the plane $z=-L$ to the plane $z=+L$ is given by:

$T=\int d t=\int \frac{d s}{c}=\int \frac{\sqrt{d \rho^{2}+d z^{2}}}{c}=\int_{-L}^{+L} \frac{d z}{c} \sqrt{1+\left(\frac{d \rho}{d z}\right)^{2}}$

This time is also equal to $2 L / v_{\text {eff }}$ leading to:

$v_{e f f}=\frac{L c}{\int_{0}^{L} d z \sqrt{1+\left(\frac{d \rho}{d z}\right)^{2}}}$

From the hyperbolic trajectories of Eq. (43), we obtain:

$$
\left(\frac{d \rho}{d z}\right)^{2}=\frac{4 \rho_{0}^{2} z^{2}}{\left.k^{2} w_{\min }^{4} z^{2}+\frac{k^{2} w_{\min }^{4}}{4}\right)}
$$

Substituting Eq. (46) into Eq. (45), the integral may be evaluated in terms of elliptic integrals. But an accurate approximation to its value is given by the following argument. When $z$ is zero, or better said close to zero, then $(d \rho / d z)^{2}$ is zero, and the average velocity of Eq. (45) is equal to $c$, corresponding to straight line trajectories parallel to the $z$-direction in the focal waist region. When $|z|$ is large, let us say twice the diffraction length $l=k w_{\min }^{2}$, the trajectories reach the asymptotic region given by $\rho(z)=2 \rho_{0} z /\left(k w_{\text {min }}^{2}\right)$ in agreement with both Eqs. (43) and (46). Now, let us consider the quantity $q=4 \rho_{0}^{2} / k^{2} w_{\min }^{4}$ appearing in Eq. (46). Using Eq. (30), the inequality $w_{\text {inc }} / f \ll 1$, already introduced in Section 3.2 , and $\rho_{0} \leq w_{\min }$, we find that $q \ll 1$ and that $(d \rho / d z)^{2}$ is small for paraxial rays. Hence, Taylor series expanding the integrand of Eq. (45) gives:

$\int_{0}^{L} d z \sqrt{1+\left(\frac{d \rho}{d z}\right)^{2}} \approx L+\frac{2 \rho_{0}^{2}}{k^{2} w_{\min }^{4}} \int_{0}^{L} d z \frac{z^{2}}{z^{2}+\frac{k^{2} w_{\min }^{4}}{4}}$

But we have:

$\int \frac{x^{2} d x}{a+b x^{2}}=\frac{x}{b}-\frac{a}{b \sqrt{a b}} \arctan x \sqrt{\frac{b}{a}}$

The integral in Eq. (47) is evaluated using Eq. (48). The argument of the arctan function at the upper limit of integration is then found to be $2 L /\left(k w_{\min }^{2}\right)$. In the laboratory, we may assume that $L$ is large enough so that the arctan is approximated to $\pi / 2$. The obtained result is then inserted in Eq. (45) leading to:

$v_{\text {eff }}=\frac{c}{1+\frac{2 \rho_{0}^{2}}{k^{2} w_{\min }^{4}}-\frac{\pi \rho_{0}^{2}}{2 k L w_{\min }^{2}}} \approx c\left[1-\frac{2 \rho_{0}^{2}}{k^{2} w_{\min }^{4}}+\frac{\pi \rho_{0}^{2}}{2 k L w_{\min }^{2}}\right]$

and to the approximation

$<v_{\text {eff }}>=c\left[1-\frac{2<\rho_{0}^{2}>}{k^{2} w_{\min }^{4}}+\frac{\pi<\rho_{0}^{2}>}{2 k L w_{\min }^{2}}\right]$

Now, $\left\langle\rho_{0}^{2}>\right.$ in the focal plane is given, analogous to Eq.
(39), by $w_{\text {inc }}^{2} / 2$. Using also Eq. (30), we then obtain:

$<v_{\text {eff }}>=c\left[1-\frac{w_{i n c}^{2}}{4 f^{2}}+\frac{\pi}{4 k L}\right]$

The term $\pi /(4 k L)$ accounts for a small increase of the average velocity due to the forward or near forward propagation in the focal region where trajectories are close to straight lines parallel to the beam forward direction. This is related to the Gouy phase advance mentioned above. Furthermore, when $L$ is large, the trajectories spend relatively more time close to the asymptotes, so that the correction term becomes an ever-smaller fraction of the total result. If this term is neglected, then we recover the usual result:

$\left.<v_{e f f}>=c \quad 1-\frac{w_{\text {inc }}^{2}}{4 f^{2}}\right)$.

3.5. Generalization of the angular spectrum approach to any shaped beam

The angular spectrum approach to Gaussian beams which has been the key to the understanding of the "less than c" effect, allows one to provide a generalization to arbitrary shaped beams. For this, we consider the field $\mathbf{E}_{\text {inc }}\left(\boldsymbol{\rho}_{\text {inc }}\right)$ incident on the focusing lens and the field $\mathbf{E}_{0}\left(\rho_{0}\right)$ at the $z=0$ focal waist plane. The Fourier transform of each of these beams is:

$$
\begin{gathered}
\mathbf{F}_{i n c}\left(\boldsymbol{\rho}_{K}\right)=\int d^{2} \rho_{\text {inc }} \mathbf{E}_{\text {inc }}\left(\rho_{\text {inc }}\right) \exp \left(-i \rho_{i n c} \boldsymbol{\rho}_{K}\right) \\
\mathbf{F}_{0}\left(\boldsymbol{\rho}_{k}\right)=\int d^{2} \rho_{0} \mathbf{E}_{0}\left(\boldsymbol{\rho}_{0}\right) \exp \left(-i \rho_{0} \rho_{k}\right)
\end{gathered}
$$

allowing us to define the mean-square widths:

$$
\begin{aligned}
& <\rho_{i n c}^{2}>=\frac{\int d^{2} \rho_{i n c} \rho_{i n c}^{2} \mathbf{E}_{i n c}^{*}\left(\rho_{i n c}\right) \cdot \mathbf{E}_{i n c}\left(\rho_{i n c}\right)}{\int d^{2} \rho_{i n c} \mathbf{E}_{i n c}^{*}\left(\rho_{i n c}\right) \cdot \mathbf{E}_{i n c}\left(\boldsymbol{\rho}_{i n c}\right)} \\
& <\rho_{K}^{2}>=\frac{\int d^{2} \rho_{K} \rho_{K}^{2} \mathbf{F}_{i n c}^{k}\left(\boldsymbol{\rho}_{K}\right) \cdot \mathbf{F}_{i n c}\left(\rho_{K}\right)}{\int d^{2} \rho_{K} \mathbf{F}_{i n c}^{*}\left(\boldsymbol{\rho}_{K}\right) \cdot \mathbf{F}_{i n c}\left(\rho_{K}\right)} \\
& <\rho_{0}^{2}>=\frac{\int d^{2} \rho_{0} \rho_{0}^{2} \mathbf{E}_{0}^{*}\left(\rho_{0}\right) \cdot \mathbf{E}_{0}\left(\rho_{0}\right)}{\int d^{2} \rho_{0} \mathbf{E}_{0}^{*}\left(\rho_{0}\right) \cdot \mathbf{E}_{0}\left(\rho_{0}\right)} \\
& <\rho_{k}^{2}>=\frac{\int d^{2} \rho_{k} \rho_{k}^{2} \mathbf{F}_{0}^{*}\left(\rho_{k}\right) \cdot \mathbf{F}_{0}\left(\rho_{k}\right)}{\int d^{2} \rho_{k} \mathbf{F}_{0}^{*}\left(\rho_{k}\right) \cdot \mathbf{F}_{0}\left(\rho_{k}\right)}
\end{aligned}
$$

The mean square widths are related by the sizebandwidth theorem in two dimensions [12]:

$$
\begin{aligned}
& <\rho_{i n c}^{2}><\rho_{K}^{2}>=L \\
& <\rho_{0}^{2}><\rho_{k}^{2}>=M
\end{aligned}
$$

where $L$ and $M$ are constants that depend on the beam shape with the Gaussian beam being the limiting case in which $L=M=1$. For other beam shapes, $L \gtrsim 1$ and $M \gtrsim 1$. Using Eqs. (34) and (60), we then have the approximation for the average velocity:

$$
\left.<v_{\text {eff }}>=c \quad 1-\frac{M}{2 k^{2}<\rho_{0}^{2}>}\right)
$$


In the paraxial limit, the incident field $\mathbf{E}_{\text {inc }}\left(\rho_{\text {inc }}\right)$ and the field $\mathbf{E}_{0}\left(\rho_{0}\right)$ at $z=0$ are related by (see p. 83-86 of [25]):

$\mathbf{E}_{0}\left(\rho_{0}\right)=\int d^{2} \rho_{i n c} \mathbf{E}_{i n c}\left(\rho_{i n c}\right) \exp \left(-i \frac{k}{f} \rho_{i n c} \rho_{0}\right)=\mathbf{F}_{i n c}\left(\frac{k}{f} \rho_{0}\right)$

meaning that the field at the center of the focal waist center is the scaled Fourier transform of the incident field $\mathbf{E}_{\text {inc }}\left(\boldsymbol{\rho}_{\text {inc }}\right)$. Then, noting that $\mathbf{F}_{i n c}=\mathbf{F}_{\text {inc }}\left(\boldsymbol{\rho}_{K}\right)$, we have $\rho_{K}=k \rho_{0} / f$, and using Eqs. (59) and (61), we obtain:

$\left.<v_{e f f}>=c 1-\frac{M}{L} \frac{1}{2} \frac{<\rho_{i n c}^{2}>}{f^{2}}\right)$

As a final step, let us consider Eq. (59) which depends on the functional forms of $\mathbf{E}_{\text {inc }}$ and $\mathbf{F}_{\text {inc }}$ and Eq. (60) which depends on the functional forms of $\mathbf{E}_{0}$ and $\mathbf{F}_{0}$. But we said (Eq. (62)) that $\mathbf{E}_{0}$ is proportional to $\mathbf{F}_{\text {inc }}$. So then, $\mathbf{F}_{0}$, which is the Fourier transform of $\mathbf{E}_{0}$, is proportional to $\mathbf{E}_{\text {inc }}$. Hence, ( $\mathbf{E}_{\text {inc }}, \mathbf{F}_{\text {inc }}$ ) and $\left(\mathbf{F}_{0}, \mathbf{E}_{0}\right)$ are the same pairs of functions, leading to $L=M$. Therefore, Eq. (63) simplifies to:

$$
\left.<v_{\text {eff }}>=c \quad 1-\frac{1}{2} \frac{<\rho_{\text {inc }}^{2}>}{f^{2}}\right)
$$

which is the generalization of the average beam velocity for any shaped beam. For a Gaussian beam for which $<\rho_{i n c}^{2}>=w_{i n c}^{2} / 2$ (see Eq. (39)), we recover Eq. (36).

\subsection{Experimental considerations}

In order to pave the way to experimental corroborations of the approaches developed above, let us consider light being emitted and propagating at the speed of light $c$ over a distance $L_{p}$ before detection. The time between emission and detection is then given by $t=L_{p} / c$. However, a light beam propagates with an average velocity $\left\langle v_{\text {eff }}\right\rangle$ and the time between emission and detection is longer, given by $t_{\text {eff }}=L_{p} /\left\langle v_{\text {eff }}\right\rangle$. The difference between these two times therefore reads:

$\Delta t=L_{p}\left(\frac{1}{\left\langle v_{e f f}\right\rangle}-\frac{1}{c}\right)$

Using Eq. (36), it is found that the time difference of propagation per unit of length of propagation reads:

$\delta t=\frac{1}{c}\left(\frac{1}{1-\frac{w_{i n c}^{2}}{4 f^{2}}}-1\right)$

Under the assumption already used that $w_{\text {inc }} / f \ll 1$, we may Taylor expand this equation to obtain:

$\delta t \approx \frac{w_{\text {inc }}^{2}}{4 c f^{2}}$

In [37], Giovannini et al. dealt with single photons propagating in a confocal telescope with focal length $f$. Two photons are generated as a photon pair strongly correlated in wavelength and generation time. The arrival times of the photons traveling two different arms of an interferometer are compared with femtosecond precision using a quantum effect known as Hong-Ou-Mandel effect.
They found that the expected spatial delay for a Gaussian beam on transmission through the confocal telescope, i.e. over a distance of propagation equal to $2 f$ reads (Eq. (2) in [37]):

$\delta z_{2 f}=\left(\frac{w_{i n c}}{f}\right)^{2} \frac{f}{2}$

Eq. (68) can be readily obtained from Eq. (67) as $\delta z_{2 f}=c \delta t_{2 f}$ in which $\delta t_{2 f}=2 f \delta t$. In their experiments, the authors of [37] used $w_{\text {inc }}=2.32 \pm 0.09 \mathrm{~mm}, f=0.40 \mathrm{~m}$. From Eq. (68), they then obtained $\delta z_{2 j}=6.7 \mu \mathrm{m}$ which compared favorably with their experimental result of $\delta z_{2 f}=7.7 \mu \mathrm{m}$.

We propose an alternative experiment relying on a classical electromagnetic set-up in which the intrinsic temporal broadening of an ultra-short light pulse during its propagation in free space would be measured. Such a "new effect" might become important when studying and using ultra-short laser pulses. To provide an order of magnitude of the effect, let us focus an incident pulse with a spatial light modulator (SLM) which would not produce any significant pulse broadening. We then use Eq. (67) which tells us that the time difference of propagation over a propagation length $L_{p}$ is $\Delta t \simeq w_{i n c}^{2} L_{p} /\left(4 c f^{2}\right)$. Let us set $L_{p}=n f$ in which $n$ is an unknown to be determined. The time difference of propagation over $L_{p}$ now reads as $\Delta t \simeq n w_{\text {inc }}^{2} /(4 c f)$. The detector is made from two parts, a second SLM which receives the pulse propagating after the focal plane and focuses it to a second part which will be used for the analysis of the pulse. The radius $w_{p}$ of the beam after a propagation length $L_{p}=2 f$ is, exactly, by symmetry, equal to the incident radius $w_{\text {inc. More gen- }}$ erally, we may evaluate the radius $w_{p}$ of the beam after a propagation length $L_{p}=n f$ as being $w_{p}=(n-1) w_{\text {inc }}$. To take full advantage of the second SLM, having the longest propagation length possible, we take $\omega_{p}=L_{D}$ in which $L_{D}$ is the largest typical dimension of the SLM. This yields $n=1+L_{D} / w_{\text {inc }}$ which does not depend on the focal length. With typical values $w_{\text {inc }}=2.5 \times 10^{-3} \mathrm{~m}$ and $L_{D}=10^{-2} \mathrm{~m}$, we obtain $n=5$. With a focal length equal to $10 w_{\text {inc }}$, i.e. $25 \mathrm{~cm}$, we obtain an intrinsic temporal broadening of the pulse equal to $100 \mathrm{fs}$. The analysis of the pulse may be carried out by using an autocorrelation measurement device [38].

\section{Failure of the optical theorem for an arbitrary beam}

\subsection{Vectorial waves}

One of the most important theorems concerning the scattering of light (and other electromagnetic radiation) is the optical theorem, also called the extinction theorem. This is not to be confused with the Ewald-Oseen extinction theorem in which the wave incident on a medium of different refractive index is extinguished, and is replaced by a new refracted wave [39]. Instead, the optical theorem states that for a wave propagating in a lossless medium and illuminating a finite scatterer, the extinction crosssection is proportional to the real part of the scattering amplitude in the forward direction $[12,13]$. In the 
framework of GLMT, this becomes:

$C_{\text {ext }}=\frac{4 \pi}{k^{2}} \operatorname{Re}\left(S_{i}(0)\right), i=1,2$

where:

$S_{i}(0)=-S_{1}(0)=-S_{2}(0)$,

and $S_{1}(0)$ and $S_{2}(0)$ are the amplitude functions of GLMT evaluated in the forward direction $\theta=0$. The minus sign is due to the convention used in GLMT to define associated Lengendre functions (see [22] for details). Let us now apply this result to the case of on-axis Gaussian beam which is defined by special BSCs according to [21]:

$g_{n X}^{m}=0, X=T E, T M,|m| \neq 1$

$$
\begin{aligned}
& g_{n, T M}^{1}=g_{n, T M}^{-1}=\frac{g_{n}}{2} \\
& g_{n, T E}^{1}=-g_{n, T E}^{-1}=-i \frac{g_{n}}{2}
\end{aligned}
$$

We then obtain (see $[22,40]$ and Eq. (21)):

$$
C_{\text {ext }}=\frac{2 \pi}{k^{2}} \operatorname{Re} \sum_{n=1}^{\infty}(2 n+1) g_{n}\left(a_{n}+b_{n}\right)
$$

which is in disagreement with Eq. (6). Therefore, the optical theorem which has been shown to be true for the case of an illuminating plane wave, e.g. $[12,13]$, is not valid for arbitrary shaped beams. An extended optical theorem for an on-axis Gaussian beam was published in [40]. This theorem takes the form of a series in powers of $s^{2}$, for which the first term has the form of Eq. (69) and the second term is $O\left(s^{2}\right)$. This is not to be confused with the generalized optical theorem which relates the imaginary part of the scattering amplitude at one angle to an integral over the product of two scattered amplitudes evaluated at other angles [41-44]. The failure of the optical theorem for Gaussian beam scattering by a spherical particle was also discussed by Lock et al. [45]. Note that these studies were carried out using the discrete expansion approach for the illuminating beam.

\subsection{Scalar waves}

This subsection is devoted to scalar waves, e.g. quantum waves or acoustical waves. The work on quantum waves summarized here was motivated by the desire to look for analogies and differences between vectorial and scalar scattering of arbitrary shaped beams. A description of arbitrary quantum beams was made in terms of free spherical waves which are eigenfunctions of the free Hamiltonian, the square of the orbital angular momentum, and the $z$-component of the orbital angular momentum. This provides a discrete expansion approach akin to Eqs. (1) and (2), in terms of two discrete subscripts and an integral over the third continuous subscript [46]. An alternative description relies on an expansion in terms of plane waves, according to:

$\psi(\mathbf{r})=\int_{\mathbf{k}} S(\mathbf{k}) \exp (i \mathbf{k} \cdot \mathbf{r}) d^{3} k$

in which $\mathbf{r}$ denotes a point in space, $\mathbf{k}$ a wavevector, $S(\mathbf{k})$ is the wavevector spectrum, and $\psi(\mathbf{r})$ is the spatial representation of the quantum state (possibly frozen, say at time $t=0$ ) [47].

In a quantum mechanical context for a plane wave, the standard equation for the optical theorem assumes the form:

$C_{e x t}^{q}=\frac{4 \pi}{k} \operatorname{Im}\left(f_{\mathbf{k}}(0)\right)$

in which $C_{\text {ext }}^{q}$ is the extinction cross-section, $q$ stands for "quantum", $k$ is a wave number, $\mathbf{k}$ is the associated wave vector, and $f_{\mathbf{k}}(0)$ is the scattering amplitude at the scattering angle $\theta=0$ associated with a radial potential $V(r)$. This equation may be rewritten as:

$C_{\text {ext }}^{q}=\frac{2 \pi}{k^{2}} \sum_{l=0}^{\infty}(2 l+1)\left(1-\operatorname{Re} S_{l}\right)$

in which $S_{i}$ 's are complex numbers with a modulus smaller than 1 , appearing in the expansion of $f_{\mathbf{k}}(\theta)$ in terms of spherical harmonics $Y_{l}^{0}(\theta)$ [48-50]. Let us now consider a wavefunction of the form given by Eq. (75). The expansion in terms of free spherical waves reads as [51]:

$\psi(\mathbf{r})=\sum_{l=0}^{\infty} \sum_{m=-l}^{+l} a_{k m m} \varphi_{k l m}^{(0)}(\mathbf{r})$

in which the spherical waves are [48]:

$\varphi_{k l m}^{(0)}(\mathbf{r})=\sqrt{\frac{2}{\pi}} k j_{l}(k r) Y_{l}^{\mathrm{m}}(\theta, \varphi)$

and the coefficients $a_{k m m}$ may be called quantum beam shape coefficients.

But it has been established that the quantum extinction cross-section for the interaction between an arbitrary quantum shaped beam of the form of Eq. (75) and a radial potential is [51]:

$C_{e x t}^{q}=\frac{1}{\pi} \sum_{l=0}^{\infty} \sum_{m=-l}^{+l}\left|a_{k l m}\right|^{2}\left(1-\operatorname{Re} S_{l}\right)$

which, in general, does not agree with the standard formula of Eq. (77). This establishes the failure of the optical theorem for arbitrary non-plane incidence in quantum mechanics.

In the same vein, Mitri and Silva used a discrete expansion approach of pressure waves in terms of BSCs (scalar counterpart of Eqs. (1) and (2)) to demonstrate the failure of the optical theorem for arbitrary non-plane wave incidence in acoustics, and to present an extended optical theorem for scalar acoustical beams of arbitrary character [52]. A similar work was later carried out in cylindrical coordinates [53].

\subsection{Insight provided by the angular spectrum approach}

The failure of the optical theorem for electromagnetic waves using the discrete expansion approach in Section 4.2 obscures the physical meaning of the extended optical theorem, and the deep reason why this failure occurs. The situation, however, becomes very clear and convincing if we rely on the angular spectrum decomposition of Eqs. (29). The optical theorem relates the extinction crosssection and a radiative property in the forward direction 
of a plane wave but, in an angular spectrum decomposition, each plane wave of the decomposition has its own forward direction of propagation which, in general, does not coincide with the forward direction of propagation of the beam, implying the failure of the optical theorem. The same argument may be used for quantum beams, invoking the angular spectrum decomposition of Eq. (75). Acoustical beams used in [52,53] may be decomposed into an angular spectrum as well, and the same argument holds as well.

\section{Conclusion}

The subject of this paper is the insight that can be obtained concerning certain aspects of beam propagation and scattering by a focused beam when considered from a complementary point of view. There have been previous derivations of the propagation speed of a transverse localized beam and the fact that the standard form of the optical theorem is violated when a transversely localized beam is scattered by a particle. But considering the incident beam as a superposition of plane waves, all having the same frequency but different propagation directions makes the two previously obtained results mentioned above in some sense obvious. Fifty years ago one of the most well-known natural philosophers of the previous generation noted that " $\ldots$ if the peculiar viewpoint taken is truly experimentally equivalent to the usual in the realm of the known, there is always a range of applications and problems in this realm for which the special viewpoint gives one special power and clarity of thought, which is valuable in itself." [54]. Concerning the insight that can be obtained by considering the problems of beam propagation and light scattering from the point of view of an angular spectrum of plane waves, we find ourselves to be in agreement with the statement quoted above.

Finally, if we insist on the preservation of the first principle mentioned in Section 1, we would have to state that a laser beam is not light. It is clearly more economical to revise the first principle by stating that a laser light is light which travels in free space slower than $c$. This statement may immediately be generalized as follows: a laser beam is light which travels in a given medium slower than the speed of a plane wave in the medium.

\section{Acknowledgments}

The authors wish to thank Dr. Gérard Gréhan of the Coria of Normandie University, Saint-Etienne du Rouvray, France, for bringing Ref. [37] to our attention. We also wish to thank Prof. Miles Padgett of the University of Glasgow for his encouragement to one of us (G.G.) at the 13th European/French Israeli Symposium on Nonlinear and Quantum Optics held in Aussois, France, 17-22 March, 2015, concerning this work. We also wish to thank Prof. Marc Brunel and Dr. Ammar Hideur for their advice in designing the classical experimental proposal.

\section{References}

[1] Epicurus. Lettres, maximes, sentences. Librairie Gẻnẻrale Française; 1994.

[2] Lucretius. De la nature (De Rerum Natura). English translation: on the nature of things. Garnier-Flammarion; 1997.

[3] Descartes. Les principes de la philosophie, troisième partie: Piroüettes et tourbillons des cieux. English translation: Principles of philosophy, part III. Paleo, France; 2000.

[4] Einstein A, Infeld L. L'évolution des idées en physique. French translation of: The evolution of physics. Petite Bibliotheque Payot; 1963.

[5] Descartes. Discours de la mthode. English translation: Discourse of the methode. Librairie philosophique J. Vrin 1938.

[6] Kant I. Critique of pure reason. Cambridge UK: Cambridge University Press; 1998.

[7] Gouesbet G. Hypotheses on the a priori rational necessity of quantum mechanics. Principia 2010;14(3):393-404

[8] Gouesbet G. Hidden worlds in quantum physics. Dover Publications, Inc; 2013.

[9] Quine WV. Le mot et la chose, French translation of word and object. Flammarion; 1977.

[10] Quine WV. La poursuite de la vérité. french translation of pursuit of truth. Paris: Editions du Seutil; 1993.

[11] Reitz JF, Milford F], Christy RW. Foundations of electromagnetic theory. thirded. MA: Addison-Wesley, Reading; 1979. 408-415.

[12] Jackson JD. Classical electrodynamics. New York: John Wiley and Sons, Inc; 1962

[13] van de Hulst HC. Light scattering by small particles. New York: Wiley; 1957.

[14] Gouesbet G, Grêhan G. Generalized Lorenz-Mie theories. Berlin: Springer; 2011.

[15] Gouesbet G, Grêhan G. Generalized Lorenz-Mie theory for assemblies of spheres and aggregates. J Opt A: Pure Appl Opt 1999;1: 706-12.

[16] Gouesbet G, Grêhan G. Generalized Lorenz-Mie theory for a sphere with an eccentrically located spherical inclusion. J Modern Opt 2000;47(5):821-37.

[17] Gouesbet G, Lock JA. On the description of electromagnetic arbitrary shaped beams. The relationship between beam shape coefficients and plane wave spectra. J Quant Spectrosc Radiat Transf 2015; 162:18-30.

[18] Gouesbet G. Partial wave expansions and properties of axisymmetric light beams. Appl Opt 1996;35(9):1543-55.

[19] Gouesbet G. T-matrix formulation and generalized Lorenz-Mie theories in spherical coordinates. Opt Commun 2010;283:517-21.

[20] Gouesbet G, Grẻhan G. Sur la généralisation de la théorie de LorenzMie. J Opt 1982;13(2):97-103.

[21] Gouesbet G, Maheu B, Grêhan G. Light scattering from a sphere arbitrarily located in a Gaussian beam, using a Bromwich formulation. J Opt Soc Am A 1988;5(9):1427-43.

[22] Gouesbet G, Grêhan G, Maheu B. Scattering of a Gaussian beam by a Mie scatter center, using a Bromwich formulation. J Opt (Paris), Republished in selected papers on light scattering. SPIE Milestone series, vol. 951; 1988, edited by M. Kerker., 1985;16(2):83-93.

[23] Colak S, Yeh C, Casperson LW. Scattering of focused beams by tenuous particles. Appl Opt 1979; 18:294-302

[24] Yeh CW, Colak S, Barber PW. Scattering of sharply focused beam by arbitrarily shaped dielectric particles: an exact solution. Appl Opt $1982 ; 21: 4426-33$

[25] Goodman JW. Introduction to Fourier optics. San Francisco: McGraw-Hill; 1968.

[26] Doicu A, Wriedt T, Eremin YA. Light scattering by systems of particles. Berlin: Springer; 2006.

[27] Lock JA. Angular spectrum and localized model of Davis-type beam. J Opt Soc Am A 2013;30(3):489-500.

[28] Neves AAR, Fontes A, Padilha LA, Rodriguez E, de Brito Cruz CH Barbosa LC, et al. Exact partial wave expansion of optical beams with respect to an arbitrary origin. Opt Lett 2006;31(16):2477-9.

[29] Richards B, Wolf E. Electromagnetic diffraction in optical systems. II Structure of the image field in an aplanatic system. Proc R Soc Lond Ser A 1959;253:358-79.

[30] Barton JP, Alexander DR. Fifth-order corrected electromagnetic field components for fundamental Gaussian beams. J Appl Phys 1989;66(7): $2800-2$

[31] Gouesbet $G$, Lock JA, Grêhan G. Partial wave representations of laser beams for use in light scattering calculations. Appl Opt 1995;34(12): 2133-43.

[32] Kogelnik H, Li T. Laser beams and resonators. Appl Opt 1966;5(10): $1550-6$. 
[33] Gouesbet G, Maheu B, Gréhan G. The order of approximation in a theory of the scattering of a Gaussian beam by a Mie scatter center. J Opt (Paris), Republished in selected papers on light scattering, SPIE Milestone series, vol. 951; 1988, edited by M. Kerker, 1985;16 (5):239-47.

[34] Spiegel MR. Theory and problems of advanced calculus, Schaum's outline series. New York: McGraw-Hill Book Company; 1963.

[35] Boyd RW. Intuitive explanations of the phase anomaly of focused light beams. J Opt Soc Am 1979;70:877-80.

[36] Born M, Wolf E. Principles of optics, sixth (corrected) ed. Cambridge, UK: Cambridge University Press; 1998

[37] Giovannini D, Romero J, Potocek V, Ferenczy G, Speirits F, Barnett SM, et al. Spatially structured photons that travel in free space slower than the speed of light. Science 2015;6224:857-60.

[38] Sala KL, Kenney-Wallace GA, Hall GE. CW autocorrelation measurements of picosecond laser pulses. IEEE J Quant Electron 1980;9: 990-6 QE-16.

[39] Born M, Wolf E. Principles of optics. sixth edCambridge UK: Cambridge University Press; 1980 Sections 2.4 .2 and 2.4.3.

[40] Gouesbet G, Letellier C, Grêhan G, Hodges JT. Generalized optical theorem for on-axis Gaussian beams. Opt Commun 1996;125: $137-57$.

[41] Newton RG. Optical theorem and beyond. Am J Phys 1976;44: $639-42$.

[42] Marston PL. Generalized optical theorem for scattering having inverse symmetries: applications to acoustic backscattering. J Acoust Soc Am 2001:109:1291-5.

[43] Zhang L, Marston PL. Axial radiation force exerted by general nondiffracting beams. J Acoust Soc Am 2012;131:329-35.
[44] Zhang L, Marston PL. Optical theorem for acoustic non-diffracting beams and application to radiation force and torque. Biomed opt Express 2013;4:1610-17, erratum 2013;4:2988.

[45] Lock JA, Hodges JT, Gouesbet G. Failure of the optical theorem for Gaussian-beam scattering by a spherical particle. J Opt Soc Am A 1995:12(12):2708-15.

[46] Gouesbet G. Expansion in free space of arbitrary quantum wavepackets, quantum laser beams, and Gaussian quantum laser beams (on-axis and off-axis) in terms of free spherical waves. Part Part Syst Charact 2005;22:38-44.

[47] Gouesbet G, Lock JA. Quantum arbitrary shaped beams revisited. Opt Commun 2007:273:296-305.

[48] Cohen-Tannoudji C, Diu B, Laloe F. Mẻcanique quantique, vols. 1 and 2.Paris: Hermann; 1973.

[49] Landau L, Lifchitz E. Mécanique quantique. Moscou: Editions Mir; 1967.

[50] Newton RG. Scattering theory of waves and particles.Mineola, NY: Dover Publications, Inc; 2002.

[51] Gouesbet $\mathrm{G}$. On the optical theorem and non-plane-wave scattering in quantum mechanics. J Math Phys 2009;50:5 Article number: 112302

[52] Mitri FG, Silva GT. Generalization of the extended optical theorem for scalar arbitrary-shape acoustical beams in spherical coordinates. Phys Rev E 2014;90 Paper 053204.

[53] Mitri FG. Optical theorem for two-dimensional (2D) scalar monochromatic acoustical beams in cylindrical coordinates. Ultrasonics 62 (2015), http://dx.doi.org/10.1016/j.ultras.2015.02.019.

[54] Feynman RP. The development of the space-time view of quantum electrodynamics. Nobel Prize in Physics Award Address, Stockholm, 11 December 1965. Science 1966;153(3737):669-708. 